\title{
Identification of Delamination in Composite Beams by Genetic Algorithm
}

\author{
M. Krawczuk and W. Ostachowicz \\ Polish Academy of Sciences \\ Institute of Fluid Flow Machinery \\ J. Fiszera 14, 80-952 Gdanisk, Poland
}

\begin{abstract}
:
This paper presents results on the identification of delamination in composite beams via genetic search technique and changes in natural frequencies. Location and size of the delamination are carried out by minimisation of an error function involving the difference between calculated and "measured" natural frequencies. Simulation studies indicate that changes in natural frequencies and genetic algorithm allow the fast and accurate estimation of delamination parameters (location and size). The advantages and limitations of the present technique are also discussed.
\end{abstract}

\section{INTRODUCTION}

The field of system identification has become an essential part of many research and application activities in the area of mechanical, aerospace and civil engineering. The increasing complexity of structures and machinery, together with the requirement for rigorous safety and reliability aspects motivates a demand for effective methods for damage locations upon and within structures.

Interest in various non-destructive damage detection methods has considerably increased over the past twenty years. During this time many methods founded on modal analysis techniques have been developed (Adams and Cawley /1/, Cawley et al. /2/, Messina et al. 13/, Krawczuk and Ostachowicz /4/, Lim and Kashangaki 15/, Farrar and Jauregui /6/). These techniques are successfully used when monitoring structures where the presence of damage leads to changes in the some of the lower natural frequencies in modal parameters.

Damage detection using changes in modal parameters has been a topic of extensive research over the past few decades $77,8 /$. Damage will cause local changes in the stiffness of the structure, which will lead to changes in its dynamic response. Changes in natural frequencies, mode shapes or amplitudes of forced vibrations are most frequently considered as damage indicators. Two distinct methodologies have been applied to identify damage parameters (location and size) in a structure using vibration data. The first method is based on finite element model updating and error localisation $19,10 \%$. The second one assumes a candidate set of possible damage scenarios (i.e. type of damage, location and size). The calculated changes in dynamic characteristics for all damage scenarios are compared to measured ones and the closest damage case is chosen $/ 2,4 /$.

Delamination, probably the most frequently occurring damage, appears as a debonding of adjoining plies in laminates composites. The causes of delamination such as imperfect bonding, crack in matrix materials, separation of adjoining plies, and broken fibres may originate during manufacturing. Alternatively, delamination may be induced during inservice loading, such as by foreign object impact or by fatigue.

This paper presents a study on the identification of delamination in composite multi-layered beams using genetic search technique and changes in natural frequencies. For the last ten years the genetic algorithm 
has become one of the most effective tools available for damage detection. The location and size of delamination are estimated by the minimisation of an output error function, which expresses the difference between calculated and measured natural frequencies. The method is demonstrated using a model of delaminated, multi-layered, composite, cantilever beam. The results obtained for simulation studies indicate the applicability of the present approach to damage detection in composite structures. Simulation studies indicate that changes in natural frequencies allow the estimation of delamination location and magnitude at high levels of accuracy and speed. The advantages and limitations of this technique are also discussed.

The paper presents a treatment of a more general case as appeared in $/ 11-15 /$. In the work described in this paper the method has been extended to the more complex inverse problem. The paper presents a deeper discussion on genetic search identification than those presented in $/ 16-18 /$. The results of this paper show that the number of calculations needed for damage detection is much less than for classical search theory.

\section{GENETIC ALGORITHM}

Genetic algorithm is a search technique based on ideas from the science of genetics and the process of natural selection. Differences between conventional search techniques and the genetic algorithm (GA) can be summarised as follows $/ 19 /$ :

- GA operates on coded form of task parameters,

- GA works with a population which represents numerical values of a particular variable,

- GA uses only objective function,

- GA applies only probabilistic rules of selection.

A simple genetic algorithm consists of three basic operations: reproduction, crossover and mutation. The algorithm starts with the randomly generated initial population. The members of this population are usually binary strings (called chromosomes). Particular elements of chromosomes are called genes. In these strings are coded values of a variable or variables, which can be a solution to examining a problem in the search space. These variables are then used to evaluate the corresponding fitness value, which is the objective function. In the next step chromosomes are selected for reproduction. The selected processes can be carried out in many ways $/ 19 /$; nevertheless the number of selected members is a function of their fitness. Thus individuals with higher fitness will receive more copies. In order to minimise the premature convergence for initial populations, special scaling methods are applied $/ 19 /$. One of the most widely applied methods is linear scaling, proposed by Bagley $/ 20 \%$. After reproduction the process of crossover is realised. There are many ways of implementing this idea $/ 19 /$. Generally crossover with one or many crossover points can be used. The crossover points are selected randomly, usually using a roulette wheel. In this way, exchanging some portions between selected chromosomes (called parents), two new strings (called children) are created. A crossover with two points is illustrated bellow. In this example two crossover points and numbers of exchanged parts of parents were randomly selected.

For:

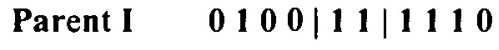

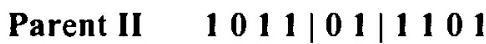

with crossover points after position 4 and 6 and crossover strings I, 1 with II, 3, next I, 2 with II, 2 and, I, 3 with II, 1: we have

\begin{tabular}{|c|c|}
\hline & 11 \\
\hline hild II & $1110|11| 0$ \\
\hline
\end{tabular}

where: I, II - denotes number of parent, whereas $1,2,3$ number of exchanged string of the parent chromosome.

The final process is mutation. Here a particular gene in a particular chromosome is randomly changed. This means that 0 is changed to a 1 , and vice versa. The process of mutation in nature is very rare and for this reason in genetic algorithm the probability of mutation in a chromosome is kept at a very low level.

\section{OBJECTIVE FUNCTION}

Objective function used in the presented paper is based on the changes in natural frequencies from "measurements". Changes in natural frequencies may be called the classical damage indicators if any. They are 
without any doubt the most used damage indicators both formerly and nowadays. The main reason for their great popularity is that natural frequencies are fairly easy to determine with a relatively high level of accuracy. In fact, one sensor placed on a structure and connected to a frequency analyser gives estimates for several natural frequencies. Further, natural frequencies are sensible to all kind of damage - local and global damage.

The form of objective function is based on a proposal by Messina et al. /3/, i.e., a Damage Location Assurance Criterion (DLAC):

$$
\operatorname{DLAC}(s)=1000 \frac{\left|\{\delta \Omega\}^{\mathrm{T}}\left\{\delta \omega_{s}\right\}\right|^{2}}{\left(\{\delta \Omega\}^{\mathrm{T}}\{\delta \Omega\} \mid\left\{\delta \omega_{s}\right\}^{\mathrm{T}}\left\{\delta \omega_{s}\right\}\right)}
$$

where $\{\delta \Omega\}$ is the trial "experimental" frequency change vector and $\left\{\delta \omega_{r}\right\}$ is the theoretical frequency change for damage at location $s$.

DLAC values lie in the range 0 to $l$, with 0 indicating no correlation and $l$ indicating an exact match between the patterns of frequency changes. The value of ( $s$ ) giving the highest DLAC values determines the predicted damage location and size. This correlation coefficient provides superior prediction success rates when compared with the Cawley-Adams algorithm /I/. The use of percentage frequency change data (rather than absolute changes) provides the best results. A problem remains when the level of damage is low. The presence of measurement error will result in a degradation of the ability to predict the damage site accurately. Nevertheless, experience shows that the method is capable of making a prediction with sufficient confidence to give a useful warning of a problem. If the predicted site is confirmed by subsequent measurements, then the method will still be seen as a valid early warning exercise.

\section{MODEL OF DELAMINATED, CANTILEVER COMPOSITE BEAM}

The model of delaminated, cantilever composite beam is presented in Fig. I.

For each part of the presented beam the following equation of transverse vibration can be written. In this

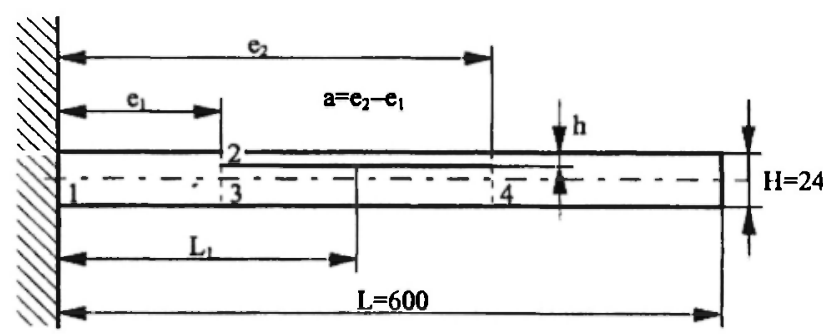

Fig. 1: Dimensions of the delaminated composite beam.

equation the shear effect is omitted.

$$
B D_{11 i} \frac{\partial^{4} y_{i}}{\partial x^{4}}+\rho A_{i} \frac{\partial^{2} y_{i}}{\partial x^{2}}=0, \quad i=1,2,3,4
$$

where: $\mathrm{B}$ denotes width of the beam, $D_{11, \mathrm{i}}$ is a bending stiffness of the beam parts, $\rho_{i}$ is a material density whereas $A_{i}$ denotes cross-sectional area of particular beam parts.

The bending stiffness for each beam parts is calculated as below:

$$
D_{11, i}=\frac{1}{3} \sum_{k=1}^{N}\left(\tilde{Q}_{11, i}\right)_{k}\left(h_{k}^{3}-h_{k-1}^{3}\right)
$$

where: $N$ is a number of laminate layers, $h_{k}$ denotes location of layer from neutral axis of the beam whereas $\left(\tilde{Q}_{11, i}\right)_{k}$ is bending stiffness of $k-t h$ layer of $i-t h$ part of the beam calculated as follows:

$$
\tilde{Q}_{11 i}=Q_{11, i} m^{4}+2\left(Q_{12 i}+2 Q_{66, i}\right) m^{2} n^{2}+Q_{22 i} n^{4}
$$

The coefficients $m$ and $n$ are equal to $m=\cos (\alpha)$ and $n=\sin (\alpha)$ respectively. $\alpha$ is an angle between fibres and neutral axis of the beam in each layer subsequently. The elements $Q_{\mathrm{ii}}(i=1,2,6, j=1,2,6)$ are calculated to take into account the following relations

$$
\left\{\begin{array}{l}
\tilde{\mathrm{Q}}_{11}=\left(1-v_{23}^{2}\right) \frac{\mathrm{E}_{11}}{\Delta} \\
\tilde{\mathrm{Q}}_{12}=v_{12}\left(1+v_{23}\right) \frac{\mathrm{E}_{22}}{\Delta} \\
\tilde{\mathrm{Q}}_{66}=\mathrm{G}_{12}
\end{array}\right.
$$


where:

$$
\Delta=1-v_{23}^{2}-2 v_{12}^{2} \frac{E_{22}}{I_{11}}-2 v_{12}^{2} v_{23} \frac{E_{22}}{I_{11}}
$$

The mechanical properties of the composite material used in equations (5-6) are calculated according to the following formulas $/ 3 /$ :

$$
\begin{aligned}
& E_{11}=E_{F} \text { vol }+E_{M}(1-v o l) \\
& E_{22}=E_{M} \frac{E_{F}+E_{M}+\left(E_{F}-E_{M}\right) \text { vol }}{E_{F}+E_{M}-\left(E_{F}-E_{M}\right) \text { vol }} \\
& v_{12}=v_{F} \text { vol }+v_{M}(1-v o l) \\
& v_{23}=v_{F} \text { vol }+v_{M}(1-v o l) \frac{1+v_{M}-v_{12} \frac{E_{M}}{E_{11}}}{1+v_{M}^{2}-v_{M} v_{12} \frac{E_{M}}{\sqrt{\varepsilon_{11}}}}
\end{aligned}
$$

where: index $F$ denotes fibres, $M$ matrix and $v o l$ is the volume fraction of fibres in the composite.

The solution of equation (2) can be expressed in the following form:

$$
\begin{aligned}
& y(x)_{i}=A_{i} \sin \left(k_{i} x\right)+B_{i} \cos \left(k_{i} x\right)+ \\
& C_{i} \sinh \left(k_{i} x\right)+D_{i} \cosh \left(k_{i} x\right) \quad i=1,4
\end{aligned}
$$

where: parameter $\mathbf{k}_{\mathbf{i}}$ corresponds with natural frequencies of the analysed beam as follows:

$$
\omega_{i}=\omega=\left(\frac{\beta_{i}}{L}\right)^{2} \sqrt{\frac{B D_{11, i}}{\rho_{i} A_{i}}} \quad k_{i}=\frac{\beta_{i}}{L} \quad i=1,2,3,4
$$

Boundary conditions for the analysed structure can be expressed in the following form:
- fixed end:

$$
\mathrm{y}_{1}(0)=0 \quad \mathrm{y}_{1}^{\prime}(0)=0
$$

- left side of delamination:

$$
\left\{\begin{array}{l}
\mathrm{y}_{1}\left(\mathrm{e}_{1}\right)=\mathrm{y}_{2}\left(\mathrm{e}_{1}\right) \mathrm{y}_{1}\left(\mathrm{e}_{1}\right)=\mathrm{y}_{3}\left(\mathrm{e}_{1}\right) \\
\mathrm{y}_{1}^{\prime}\left(\mathrm{e}_{1}\right)=\mathrm{y}_{2}^{\prime}\left(\mathrm{e}_{1}\right) \mathrm{y}_{1}^{\prime}\left(\mathrm{e}_{1}\right)=\mathrm{y}_{3}^{\prime}\left(\mathrm{e}_{1}\right) \\
\mathrm{D}_{1} \mathrm{y}_{1}^{\prime \prime}\left(\mathrm{e}_{1}\right)-\mathrm{D}_{2} \mathrm{y}_{2}^{\prime \prime}\left(\mathrm{e}_{1}\right)-\mathrm{D}_{3} \mathrm{y}_{3}^{\prime \prime}\left(\mathrm{e}_{1}\right)=0 \\
\mathrm{D}_{1} \mathrm{y}_{1}^{\prime \prime}\left(\mathrm{e}_{1}\right)-\mathrm{D}_{2} \mathrm{y}_{2}^{\prime \prime}\left(\mathrm{e}_{1}\right)-\mathrm{D}_{3} \mathrm{y}_{3}^{\prime \prime}\left(\mathrm{e}_{1}\right)=0
\end{array}\right.
$$

- right side of delamination:

$$
\begin{aligned}
& \left\{\begin{array}{l}
\mathrm{y}_{1}\left(\mathrm{e}_{2}\right)=\mathrm{y}_{2}\left(\mathrm{e}_{2}\right) \quad \mathrm{y}_{1}\left(\mathrm{e}_{2}\right)=\mathrm{y}_{3}\left(\mathrm{e}_{2}\right) \\
\mathrm{y}_{1}^{\prime}\left(\mathrm{e}_{2}\right)=\mathrm{y}_{2}^{\prime}\left(\mathrm{e}_{2}\right) \mathrm{y}_{1}^{\prime}\left(\mathrm{e}_{2}\right)=\mathrm{y}_{3}^{\prime}\left(\mathrm{e}_{2}\right) \\
\mathrm{D}_{4} \mathrm{y}_{1}^{\prime \prime}\left(\mathrm{e}_{2}\right)-\mathrm{D}_{2} \mathrm{y}_{2}^{\prime \prime}\left(\mathrm{e}_{2}\right)-\mathrm{D}_{3} \mathrm{y}_{3}^{\prime \prime}\left(\mathrm{e}_{2}\right)=0 \\
\mathrm{D}_{4} \mathrm{y}_{1}^{\prime \prime}\left(\mathrm{e}_{2}\right)-\mathrm{D}_{2} \mathrm{y}_{2}^{\prime \prime}\left(\mathrm{e}_{2}\right)-\mathrm{D}_{3} \mathrm{y}_{3}^{\prime \prime}\left(\mathrm{e}_{2}\right)=0
\end{array}\right. \\
& \text {-free end: } \\
& \qquad \mathrm{y}_{4}^{\prime \prime}(\mathrm{L})=0 \quad \mathrm{y}_{4}^{\prime \prime}(\mathrm{L})=0
\end{aligned}
$$

Taking into account boundary conditions and form of the solution of equation of motion (2) the characteristic equation of the problem can be formulated. This equation allows determination of the natural frequencies of the delaminated, cantilever, multi-layered composite beam.

$$
\operatorname{det}\left[\begin{array}{cccc}
A_{1} & 0 & 0 & 0 \\
B_{1} & B_{2} & B_{3} & 0 \\
0 & C_{2} & C_{3} & C_{4} \\
0 & 0 & 0 & D_{4}
\end{array}\right]=0
$$

where:

$$
A_{1}=\left[\begin{array}{llll}
0 & 1 & 0 & 1 \\
k_{1} & 0 & k_{1} & 0
\end{array}\right]
$$

$$
\mathrm{B}_{1}=\left[\begin{array}{lcll}
\sin \left(\mathrm{k}_{1} \mathrm{e}_{1}\right) & \cos \left(\mathrm{k}_{1} \mathrm{e}_{1}\right) & \operatorname{sh}\left(\mathrm{k}_{1} \mathrm{e}_{1}\right) & \operatorname{ch}\left(\mathrm{k}_{1} \mathrm{e}_{1}\right) \\
\sin \left(\mathrm{k}_{1} \mathrm{e}_{1}\right) & \cos \left(\mathrm{k}_{1} \mathrm{e}_{1}\right) & \operatorname{sh}\left(\mathrm{k}_{1} \mathrm{e}_{1}\right) & \operatorname{ch}\left(\mathrm{k}_{1} \mathrm{e}_{1}\right) \\
\mathrm{k}_{1} \cos \left(\mathrm{k}_{1} \mathrm{e}_{1}\right) & -\mathrm{k}_{1} \sin \left(\mathrm{k}_{1} \mathrm{e}_{1}\right) & \mathrm{k}_{1} \operatorname{ch}\left(\mathrm{k}_{1} \mathrm{e}_{1}\right) & \mathrm{k}_{1} \operatorname{sh}\left(\mathrm{k}_{1} \mathrm{e}_{1}\right) \\
\mathrm{k}_{1} \cos \left(\mathrm{k}_{1} \mathrm{e}_{1}\right) & -\mathrm{k}_{1} \sin \left(\mathrm{k}_{1} \mathrm{e}_{1}\right) & \mathrm{k}_{1} \operatorname{ch}\left(\mathrm{k}_{1} \mathrm{e}_{1}\right) & \mathrm{k}_{1} \operatorname{sh}\left(\mathrm{k}_{1} \mathrm{e}_{1}\right) \\
-\mathrm{k}_{1}{ }^{2} \sin \left(\mathrm{k}_{1} \mathrm{e}_{1}\right) & -\mathrm{k}_{1}{ }^{2} \cos \left(\mathrm{k}_{1} \mathrm{e}_{1}\right) & \mathrm{k}_{1}{ }^{2} \operatorname{sh}\left(\mathrm{k}_{1} \mathrm{e}_{1}\right) & \mathrm{k}_{1}{ }^{2} \operatorname{ch}\left(\mathrm{k}_{1} \mathrm{e}_{1}\right) \\
-\mathrm{k}_{1}{ }^{3} \cos \left(\mathrm{k}_{1} \mathrm{e}_{1}\right) & \mathrm{k}_{1}{ }^{3} \sin \left(\mathrm{k}_{1} \mathrm{e}_{1}\right) & \mathrm{k}_{1}{ }^{3} \operatorname{ch}\left(\mathrm{k}_{1} \mathrm{e}_{1}\right) & \mathrm{k}_{1}{ }^{3} \operatorname{sh}\left(\mathrm{k}_{1} \mathrm{e}_{1}\right)
\end{array}\right]
$$


$\mathrm{B}_{2}=\left[\begin{array}{llll}-\sin \left(\mathrm{k}_{2} \mathrm{e}_{1}\right) & -\cos \left(\mathrm{k}_{2} \mathrm{e}_{1}\right) & -\operatorname{sh}\left(\mathrm{k}_{2} \mathrm{e}_{1}\right) & -\operatorname{ch}\left(\mathrm{k}_{2} \mathrm{e}_{1}\right) \\ 0 & 0 & 0 & 0 \\ -\mathrm{k}_{2} \cos \left(\mathrm{k}_{2} \mathrm{e}_{1}\right) & \mathrm{k}_{2} \sin \left(\mathrm{k}_{2} \mathrm{e}_{1}\right) & -\mathrm{k}_{2} \operatorname{ch}\left(\mathrm{k}_{2} \mathrm{e}_{1}\right) & -\mathrm{k}_{2} \operatorname{sh}\left(\mathrm{k}_{2} \mathrm{e}_{1}\right) \\ 0 & 0 & 0 & 0 \\ \mathrm{k}_{2}{ }^{2} \sin \left(\mathrm{k}_{2} \mathrm{e}_{1}\right) \frac{\mathrm{D}_{2}}{\mathrm{D}_{1}} & \mathrm{k}_{2}{ }^{2} \cos \left(\mathrm{k}_{2} \mathrm{e}_{1}\right) \frac{\mathrm{D}_{2}}{\mathrm{D}_{1}} & -\mathrm{k}_{2}{ }^{2} \operatorname{sh}\left(\mathrm{k}_{2} \mathrm{e}_{1}\right) \frac{\mathrm{D}_{2}}{\mathrm{D}_{1}} & -\mathrm{k}_{2}{ }^{2} \operatorname{ch}\left(\mathrm{k}_{2} \mathrm{e}_{1}\right) \frac{\mathrm{D}_{2}}{\mathrm{D}_{1}} \\ \mathrm{k}_{2}{ }^{3} \cos \left(\mathrm{k}_{2} \mathrm{e}_{1}\right) \frac{\mathrm{D} !}{\mathrm{D}_{1}} & -\mathrm{k}_{2}{ }^{3} \sin \left(\mathrm{k}_{2} \mathrm{e}_{1}\right) \frac{\mathrm{D}_{2}}{\mathrm{D}_{1}} & -\mathrm{k}_{2}{ }^{3} \operatorname{ch}\left(\mathrm{k}_{2} \mathrm{e}_{1}\right) \frac{\mathrm{D}_{2}}{\mathrm{D}_{1}} & -\mathrm{k}_{2}{ }^{3} \operatorname{sh}\left(\mathrm{k}_{2} \mathrm{e}_{1}\right) \frac{\mathrm{D}_{2}}{\mathrm{D}_{1}}\end{array}\right]$

$\mathrm{B}_{3}=\left[\begin{array}{llll}0 & 0 & 0 & 0 \\ -\sin \left(\mathrm{k}_{3} \mathrm{e}_{1}\right) & -\cos \left(\mathrm{k}_{3} \mathrm{e}_{1}\right) & -\operatorname{sh}\left(\mathrm{k}_{3} \mathrm{e}_{1}\right) & -\operatorname{ch}\left(\mathrm{k}_{3} \mathrm{e}_{1}\right) \\ 0 & 0 & 0 & 0 \\ -\mathrm{k}_{3} \cos \left(\mathrm{k}_{3} \mathrm{e}_{1}\right) & \mathrm{k}_{3} \sin \left(\mathrm{k}_{3} \mathrm{e}_{1}\right) & -\cdot \mathrm{k}_{3} \operatorname{ch}\left(\mathrm{k}_{3} \mathrm{e}_{1}\right) & -\mathrm{k}_{3} \operatorname{sh}\left(\mathrm{k}_{3} \mathrm{e}_{1}\right) \\ \mathrm{k}_{3}{ }^{2} \sin \left(\mathrm{k}_{3} \mathrm{e}_{1}\right) \frac{\mathrm{D}_{3}}{\mathrm{D}_{1}} & \mathrm{k}_{3}{ }^{2} \cos \left(\mathrm{k}_{3} \mathrm{e}_{1}\right) \frac{\mathrm{D}_{3}}{\mathrm{D}_{1}} & --\mathrm{k}_{3}{ }^{2} \operatorname{sh}\left(\mathrm{k}_{3} \mathrm{e}_{1}\right) \frac{\mathrm{D}_{3}}{\mathrm{D}_{1}} & -\mathrm{k}_{3}{ }^{2} \operatorname{ch}\left(\mathrm{k}_{3} \mathrm{e}_{1}\right) \frac{\mathrm{D}_{3}}{\mathrm{D}_{1}} \\ \mathrm{k}_{3}{ }^{3} \cos \left(\mathrm{k}_{3} \mathrm{e}_{1}\right) \frac{\mathrm{D}_{3}}{\mathrm{D}_{1}} & -\mathrm{k}_{3}{ }^{2} \sin \left(\mathrm{k}_{3} \mathrm{e}_{1}\right) \frac{\mathrm{D}_{3}}{\mathrm{D}_{1}} & -\mathrm{k}_{3}{ }^{3} \operatorname{ch}\left(\mathrm{k}_{3} \mathrm{e}_{1}\right) \frac{\mathrm{D}_{3}}{\mathrm{D}_{1}} & -\mathrm{k}_{3}{ }^{3} \operatorname{sh}\left(\mathrm{k}_{3} \mathrm{e}_{1}\right) \frac{\mathrm{D}_{3}}{\mathrm{D}_{1}}\end{array}\right]$

$\mathrm{C}_{2}=\left[\begin{array}{llll}\sin \left(\mathrm{k}_{1} \mathrm{e}_{2}\right) & \cos \left(\mathrm{k}_{1} \mathrm{e}_{2}\right) & \operatorname{sh}\left(\mathrm{k}_{1} \mathrm{e}_{2}\right) & \operatorname{ch}\left(\mathrm{k}_{1} \mathrm{e}_{2}\right) \\ \sin \left(\mathrm{k}_{1} \mathrm{e}_{2}\right) & \cos \left(\mathrm{k}_{1} \mathrm{e}_{2}\right) & \operatorname{sh}\left(\mathrm{k}_{1} \mathrm{e}_{2}\right) & \operatorname{ch}\left(\mathrm{k}_{1} \mathrm{e}_{2}\right) \\ \mathrm{k}_{1} \cos \left(\mathrm{k}_{1} \mathrm{e}_{2}\right) & -\mathrm{k}_{1} \sin \left(\mathrm{k}_{1} \mathrm{e}_{2}\right) & \mathrm{k}_{1} \operatorname{ch}\left(\mathrm{k}_{1} \mathrm{e}_{2}\right) & \mathrm{k}_{1} \operatorname{sh}\left(\mathrm{k}_{1} \mathrm{e}_{2}\right) \\ \mathrm{k}_{1} \cos \left(\mathrm{k}_{1} \mathrm{e}_{2}\right) & -\mathrm{k}_{1} \sin \left(\mathrm{k}_{1} \mathrm{e}_{2}\right) & \mathrm{k}_{1} \operatorname{ch}\left(\mathrm{k}_{1} \mathrm{e}_{2}\right) & \mathrm{k}_{1} \operatorname{sh}\left(\mathrm{k}_{1} \mathrm{e}_{2}\right) \\ -\mathrm{k}_{1}{ }^{2} \sin \left(\mathrm{k}_{1} \mathrm{e}_{2}\right) & -\mathrm{k}_{1}{ }^{2} \cos \left(\mathrm{k}_{1} \mathrm{e}_{2}\right) & \mathrm{k}_{1}{ }^{2} \operatorname{sh}\left(\mathrm{k}_{1} \mathrm{e}_{2}\right) & \mathrm{k}_{1}{ }^{2} \operatorname{ch}\left(\mathrm{k}_{1} \mathrm{e}_{2}\right) \\ -\mathrm{k}_{1}{ }^{2} \cos \left(\mathrm{k}_{1} \mathrm{e}_{2}\right) & \mathrm{k}_{1}{ }^{3} \sin \left(\mathrm{k}_{1} \mathrm{e}_{2}\right) & \mathrm{k}_{1}{ }^{2} \operatorname{ch}\left(\mathrm{k}_{1} \mathrm{e}_{2}\right) & \mathrm{k}_{1}{ }^{2} \operatorname{sh}\left(\mathrm{k}_{1} \mathrm{e}_{2}\right)\end{array}\right]$

$\mathrm{C}_{3}=\left[\begin{array}{llll}-\sin \left(\mathrm{k}_{2} \mathrm{e}_{2}\right) & -\cos \left(\mathrm{k}_{2} \mathrm{e}_{2}\right) & -\operatorname{sh}\left(\mathrm{k}_{2} \mathrm{e}_{2}\right) & -\operatorname{ch}\left(\mathrm{k}_{2} \mathrm{e}_{2}\right) \\ 0 & 0 & 0 & 0 \\ -\mathrm{k} \cos \left(\mathrm{k}_{2} \mathrm{e}_{2}\right) & \mathrm{k}_{2} \sin \left(\mathrm{k}_{2} \mathrm{e}_{2}\right) & -\mathrm{k}_{2} \operatorname{ch}\left(\mathrm{k}_{2} \mathrm{e}_{2}\right) & -\mathrm{ksh}\left(\mathrm{k}_{2} \mathrm{e}_{2}\right) \\ 0 & 0 & 0 & 0 \\ \mathrm{k}_{2}{ }^{2} \sin \left(\mathrm{k}_{2} \mathrm{e}_{2}\right) \frac{\mathrm{D}_{2}}{\mathrm{D}_{4}} & \mathrm{k}_{2}{ }^{2} \cos \left(\mathrm{k}_{2} \mathrm{e}_{2}\right) \frac{\mathrm{D}_{2}}{\mathrm{D}_{4}} & -\mathrm{k}_{2}{ }^{3} \sin \left(\mathrm{k}_{2} \mathrm{e}_{2}\right) \frac{\mathrm{D}_{2}}{\overline{\mathrm{D}}_{4}} & -\mathrm{k}_{2}{ }^{2} \operatorname{ch}\left(\mathrm{k}_{2} \mathrm{e}_{2}\right) \frac{\mathrm{D}_{2}}{\overline{\mathrm{D}}_{4}} \\ \mathrm{k}_{2}{ }^{3} \cos \left(\mathrm{k}_{2} \mathrm{e}_{2}\right) \frac{\mathrm{D}_{2}}{\mathrm{D}_{4}} & -\mathrm{k}_{2}{ }^{3} \sin \left(\mathrm{k}_{2} \mathrm{e}_{2}\right) \frac{\mathrm{D}_{2}}{\mathrm{D}_{4}} & -\mathrm{k}_{2}{ }^{3} \operatorname{ch}\left(\mathrm{k}_{2} \mathrm{e}_{2}\right) \frac{\mathrm{D}_{2}}{\mathrm{D}_{4}} & -\mathrm{k}_{2}{ }^{3} \operatorname{sh}\left(\mathrm{k}_{2} \mathrm{e}_{2}\right) \frac{\mathrm{D}_{2}}{\mathrm{D}_{4}}\end{array}\right]$

$\mathrm{C}_{4}=\left[\begin{array}{llll}0 & 0 & 0 & 0 \\ -\sin \left(\mathrm{k}_{3} \mathrm{e}_{2}\right) & -\cos \left(\mathrm{k}_{3} \mathrm{e}_{2}\right) & -\operatorname{sh}\left(\mathrm{k}_{3} \mathrm{e}_{2}\right) & -\operatorname{ch}\left(\mathrm{k}_{3} \mathrm{e}_{2}\right) \\ 0 & 0 & 0 & 0 \\ -\mathrm{k}_{3} \cos \left(\mathrm{k}_{3} \mathrm{e}_{2}\right) & \mathrm{k}_{3} \sin \left(\mathrm{k}_{3} \mathrm{e}_{2}\right) & -\mathrm{k}_{3} \operatorname{ch}\left(\mathrm{k}_{3} \mathrm{e}_{2}\right) & -\mathrm{k}_{3} \operatorname{sh}\left(\mathrm{k}_{3} \mathrm{e}_{2}\right) \\ \mathrm{k}_{3}{ }^{2} \sin \left(\mathrm{k}_{3} \mathrm{e}_{2}\right) \frac{\mathrm{D}_{3}}{\mathrm{D}_{4}} & \mathrm{k}_{3}{ }^{2} \cos \left(\mathrm{k}_{3} \mathrm{e}_{2}\right) \frac{\mathrm{D}_{3}}{\mathrm{D}_{4}} & -\mathrm{k}_{3}{ }^{2} \operatorname{sh}\left(\mathrm{k}_{3} \mathrm{e}_{2}\right) \frac{\mathrm{D}_{3}}{\mathrm{D}_{4}} & -\mathrm{k}_{3}{ }^{2} \operatorname{ch}\left(\mathrm{k}_{3} \mathrm{e}_{2}\right) \frac{\mathrm{D}_{3}}{\mathrm{D}_{4}} \\ \mathrm{k}_{3}{ }^{3} \cos \left(\mathrm{k}_{3} \mathrm{e}_{2}\right) \frac{\mathrm{D}_{3}}{\bar{D}_{4}} & -\mathrm{k}_{3}{ }^{3} \sin \left(\mathrm{k}_{3} \mathrm{e}_{2}\right) \frac{\mathrm{D}_{3}}{\mathrm{D}_{4}} & -\mathrm{k}_{3}{ }^{3} \operatorname{ch}\left(\mathrm{k}_{3} \mathrm{e}_{2}\right) \frac{\mathrm{D}_{3}}{\mathrm{D}_{4}} & -\mathrm{k}_{3}{ }^{3} \operatorname{sh}\left(\mathrm{k}_{3} \mathrm{e}_{2}\right) \frac{\mathrm{D}_{3}}{\mathrm{D}_{4}}\end{array}\right]$

$\mathrm{D}_{4}=\left[\begin{array}{cccc}-\mathrm{k}_{4}{ }^{2} \sin \left(\mathrm{k}_{4} \mathrm{~L}\right) & -\mathrm{k}_{4}{ }^{3} \cos \left(\mathrm{k}_{4} \mathrm{~L}\right) & \mathrm{k}_{4}{ }^{2} \operatorname{sh}\left(\mathrm{k}_{4} \mathrm{~L}\right) & \mathrm{k}_{4}{ }^{2} \operatorname{ch}\left(\mathrm{k}_{4} \mathrm{~L}\right) \\ -\cdot \mathrm{k}_{4}{ }^{3} \cos \left(\mathrm{k}_{4} \mathrm{~L}\right) & \mathrm{k}_{4}{ }^{3} \sin \left(\mathrm{k}_{4} \mathrm{~L}\right) & \mathrm{k}_{4}{ }^{3} \operatorname{ch}\left(\mathrm{k}_{4} \mathrm{~L}\right) & \mathrm{k}_{4}{ }^{3} \operatorname{sh}\left(\mathrm{k}_{4} \mathrm{~L}\right)\end{array}\right]$

From the above equation it arises that changes in natural frequencies due to delaminations will be functions of the length of delamination, their location along the beam length and also along the beam height. 


\section{NUMERICAL EXAMPLES}

The numerical calculations were performed for cantilever, delaminated composite beam. The length of beam was equal to $0.4 \mathrm{~m}$, width $0.02 \mathrm{~m}$ and height 0.012 $m$. The beam had twelve layers $(+45 /-45)$ glass-epoxy (see also Table 1) composite material. The volume fraction of glass fibers in the analyzed beam was equal to $30 \%$.

Table 1.

Material properties of composite material components.

\begin{tabular}{|l|l|l|}
\hline & Matrix-Epoxy & Fibers-Glass \\
\hline $\begin{array}{l}\text { Young's } \\
\text { modulus }\end{array}$ & $\mathrm{E}_{\mathrm{m}}=3.43 \mathrm{GPa}$ & $\mathrm{E}_{\mathrm{f}}=66.5 \mathrm{GPa}$ \\
\hline Poisson ratio & $v_{\mathrm{m}}=0.35$ & $v_{\mathrm{f}}=0.23$ \\
\hline $\begin{array}{l}\text { Kirchoff } \\
\text { modulus }\end{array}$ & $\mathrm{G}_{\mathrm{m}}=1.27 \mathrm{GPa}$ & $\mathrm{G}_{\mathrm{f}}=27.0 \mathrm{GPa}$ \\
\hline Density & $\rho_{\mathrm{m}}=1250 \mathrm{~kg} / \mathrm{m}^{3}$ & $\rho_{\mathrm{f}}=2250 \mathrm{~kg} / \mathrm{m}^{3}$ \\
\hline
\end{tabular}

Two cases were tested:

Case 1 - the delamination had a length equal to $35 \%$ of total beam length, and was located between 6 and 7 layer (neutral axis of the beam). The left end of the delamination was located $0.1 \mathrm{~m}$ from the fixed end $\left(e_{1}=L_{I} / L=0.25\right)$, whereas the right end of the delamination was located $0.24 \mathrm{~m}$ from the fixed end $\left(e_{2}=L 2 / L=0.6\right)$.

Case 2 - the delamination had a length equal to $15 \%$ of total beam length, and was located between 4 and 5 layer. The left end of the delamination was located 0.28 $m$ from the fixed end $\left(e_{l}=L_{1} / L=0.7\right)$, whereas right end of the delamination was located $0.34 \mathrm{~m}$ from the fixed end $\left(e_{2}=L / 2 / L=0.85\right)$.

In all cases the population had 6 members. One member had 33 bits (11 for each variable). During numerical calculations it was assumed that probability of crossover is $95 \%$ and probability of mutation is $0.05 \%$. The delamination length and location were identified using the eigen sensitivity approach described in point 3. The first four natural frequencies were used in numerical tests. Only one run of the genetic algorithm was used for each case. Results of numerical calculations are presented in Figs. 2-9.

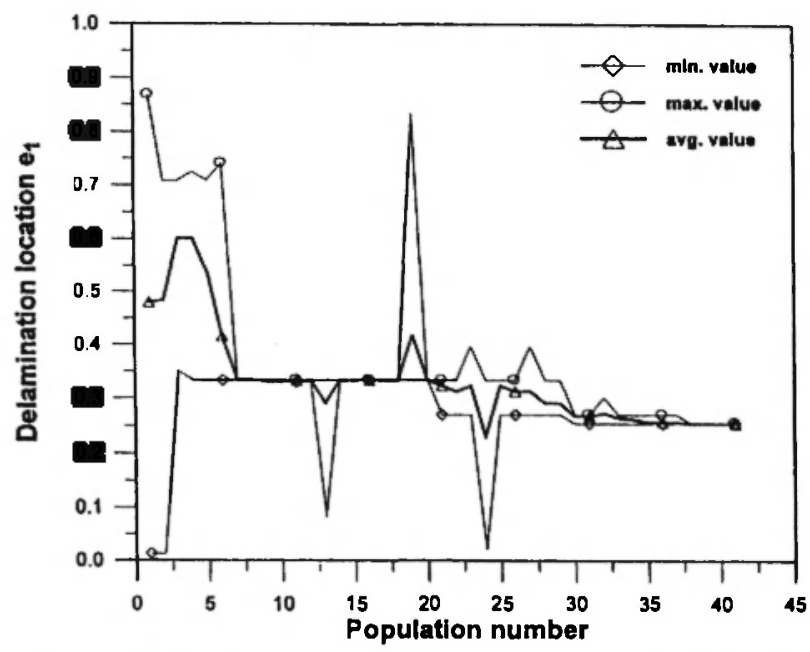

Fig. 2: Delamination location $e_{1}$ as a function of number of population - case 1 .

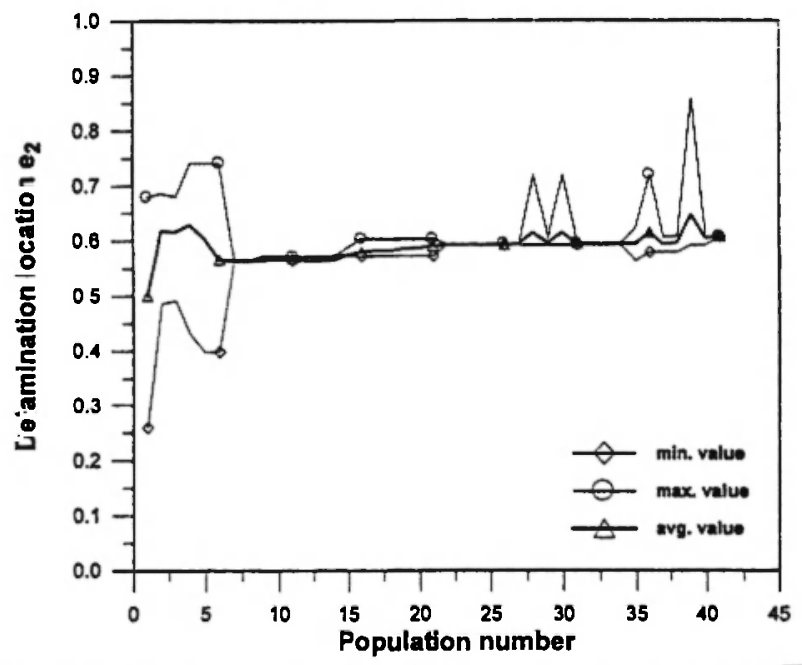

Fig. 3: Delamination location $e_{2}$ as a function of number of population - case 1 .

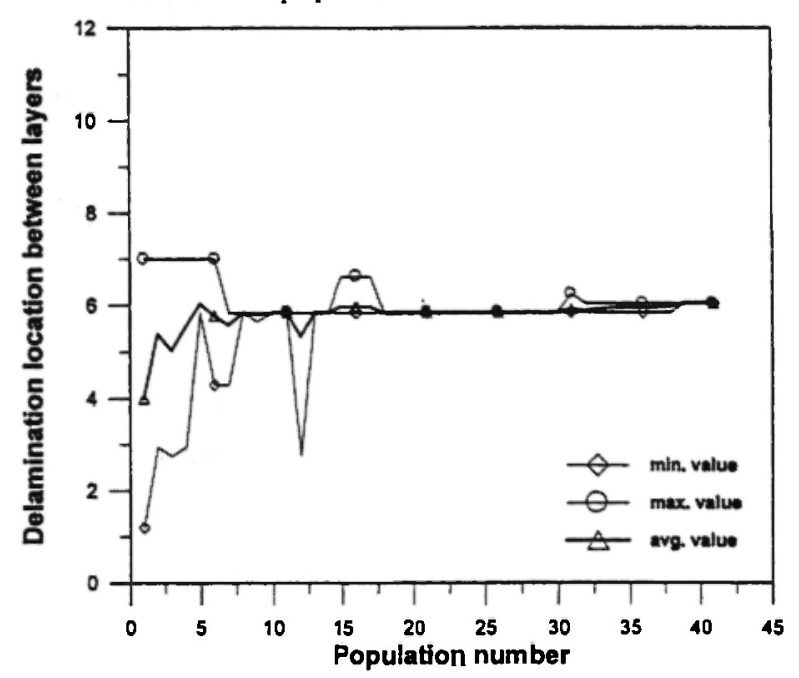

Fig. 4: Delamination location between layers as a function of number of population - case 1 . 


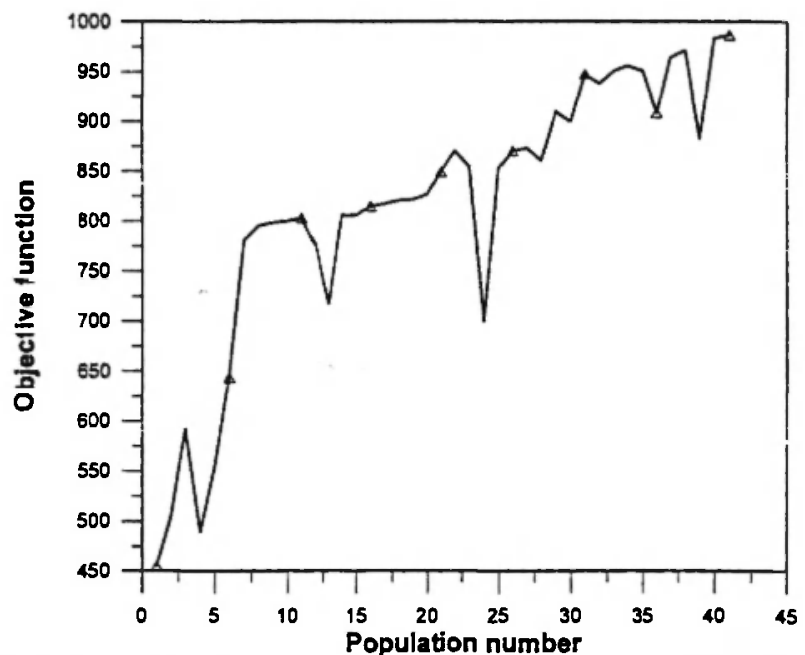

Fig. 5: Objective function as a function of number of population - case 1 .

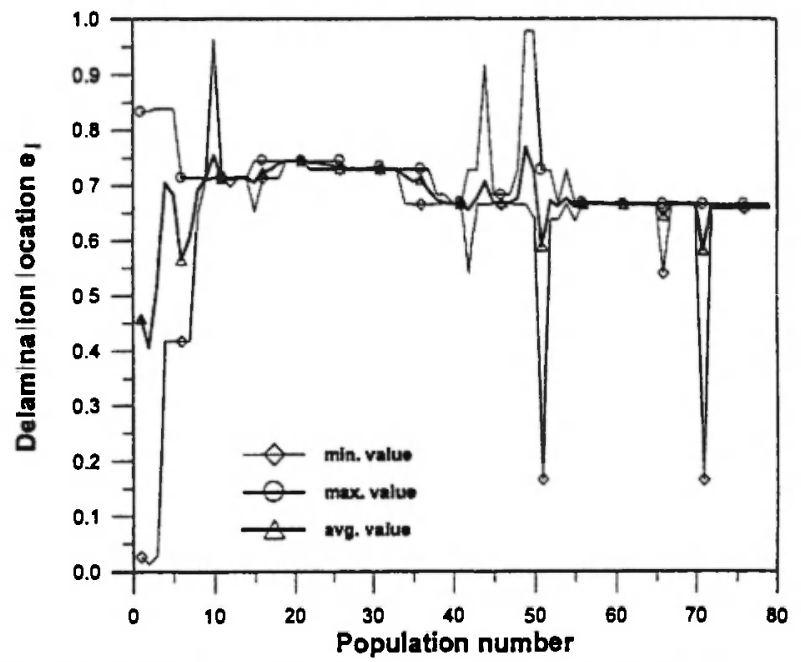

Fig. 6: Delamination location $\mathrm{e}_{1}$ as a function of number of population - case 2 .

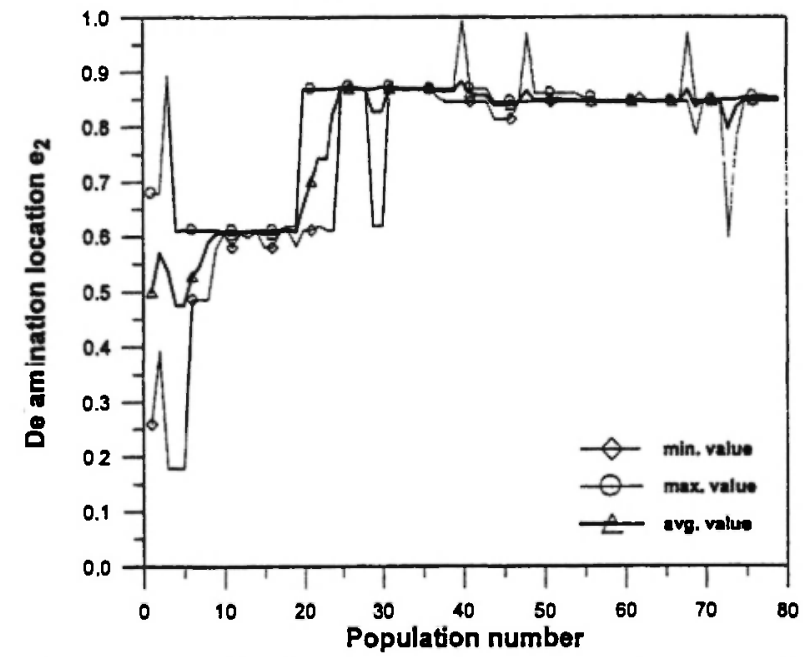

Fig. 7: Delamination location $e_{2}$ as a function of number of population - case 2 .

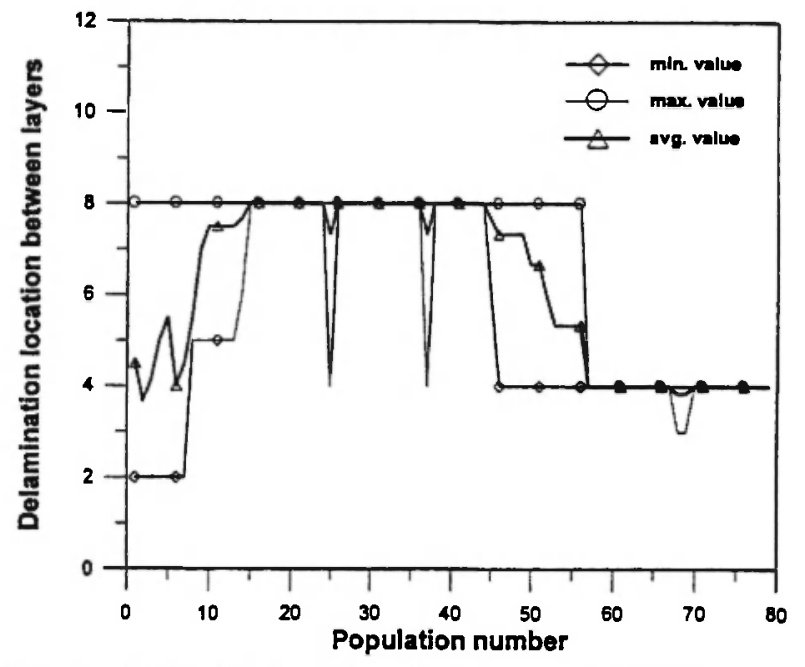

Fig. 8: Delamination location between layers as a function of number of population- case 2 .

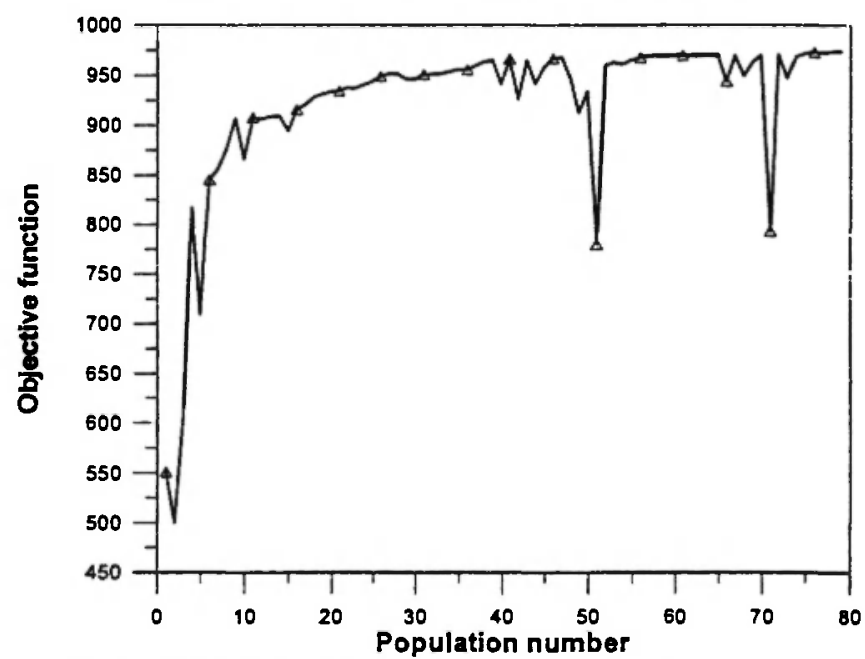

Fig. 9: Objective function as a function of number of population - case 2 .

From Figs. 2-9 it is seen that the genetic algorithm correctly locates the damage and also correctly estimates its size. The convergence to proper results was obtained after no more than 45 populations in the first case and 80 populations in case number 2 .

\section{CONCLUSIONS}

A combined genetic algorithm and eigensensitivity criterion DLAC as an objective function has been used to identify location and size of delamination from "experimental" vibration data. The genetic algorithm 
presented in this paper is very simple. Nevertheless the results obtained are promising. The number of calculations needed for damage detection is much less than for classical search algorithms. For this reason the time of numerical calculations is shorter.

Future works should be devoted to implementation in this algorithm of processes that are observed in nature. For example elitism, where the best solution is always passed on to the next generation, is a particular feature for which good results have been reported $/ 7 /$. It is also planned to check other vibration criteria applied in structural health monitoring based on changes in mode shapes and amplitudes of forced vibrations as objective functions. Such comparative analysis should explain which damage indicator is most sensitive to changes in stiffness of the structure due to damage.

\section{REFERENCES}

1. R.D. Adams and P. Cawley. The localisation of defects in structures from measurements of natural frequencies. Journal of Strain Analysis, 14(2), 4957 (1979).

2. P. Cawley, R.D. Adams, C.J. Pye and B.J. Stone. A vibration technique for non-destructively assessing the integrity of structures. Journal of Mechanical Engineering Sciences; 20(2), 93-100 (1978).

3. A. Messina, I.A. Jones and E.J. Williams. Damage detection and localisation using natural frequency changes. Proceedings of the $1^{s t}$ Conference on Identification, Cambridge, 67-76, 1992.

4. M. Krawczuk and W. Ostachowicz. Damage indicators for diagnostic of fatigue cracks in structures by vibration measurements - a survey. Journal of Theoretical and Applied Mechanics; 34(2), 307-326 (1996).

5. T.W. Lim and T.A.L. Kashangaki. Structural damage detection of space truss structures using best achievable eigenvectors. AIAA Journal, 30(9), 2310-2317 (1994).

6. C.R. Farrar and D. Jauregui. Damage detection algorithms applied to experimental and numerical modal data from 1-40 bridge. Report No. LA13074, Los Alamos National Laboratory, Los
Alamos, NM., 1996.

7. Structural Health Monitoring: Current Status and Perspectives, edited by Fu-Kuo Chang, Technomic Pub., Basel, 1997.

8. Structural Health Monitoring: Current Status and Perspectives, edited by Fu-Kuo Chang, Technomic Pub., Basel (1999).

9. M.I. Friswell and J.E. Mottershead. Finite Element Model Updating in Structural Dynamics, Kluwer Academic Publishers, 1995.

10. M. Kaouk and D.C. Zimmerman. Evaluation of the minimum rank update in damage detection: an experimental study. $1 l^{\text {th }}$ International Modal Analysis Conference, Kissimmee, Florida, 10611068, 1993.

11. P.M. Majumdar and S. Suryanarayan. Flexural Vibrations of beams with delaminations. Journal of Sound and Vibration 125, 441-461 (1988).

12. J.J. Tracy and G.C. Pardoen. Effect of delamination on the natural frequencies of composite laminates. Journal of Composite Materials 23, 1200-1215 (1989).

13. M.H.H. Shen and J.E. Grady. Free vibration of delaminated beams. AIAA Journal 30, 1361-1370 (1992).

14. B.V.A. Sankar. Finite element for modelling delamination in composite beams. Computers and Structures 38, 239-246 (1991).

15. M. Krawczuk, W. Ostachowicz and A. Zak Dynamic of cracked composite material structures. Computational Mechanics 20, 79-83 (1997).

16. M. Krawczuk, and W. Ostachowicz. Spectral finite element and genetic algorithm for crack detection in cantilever rod. Key Engineering Materials, 204205, 241-250 (2001)

17. M. Krawczuk and W. Ostachowicz. Spectral beam finite element and genetic algorithm for crack detection in beams. Proceedings of the 3rd International Workshop on Structural Health Monitoring, Stanford, CA, USA, 12-14 September, 889-898, 2001.

18. W. Ostachowicz, M. Krawczuk and M. Cartmell. The location of a concentrated mass on rectangular plates from measurements of natural vibrations. Computers and Structures (in press), (2002).

19. D. Goldberg. Genetic Algorithm in Search, 
Optimization and Machine Learning. AddisonWesley Publishing Company, Reading, 1989.

20. J.D. Bagley. The Behavior Adaptive Systems which Employ Genetic and Correlation Algorithms. PhD thesis, University of Michigan (1967). 
